\title{
Central limit theorem and almost sure results for the empirical estimator of superquantiles/CVaR in the stationary case
}

\author{
Jérôme Dedecker, Florence Merlevède ${ }^{\dagger}$
}

May 2, 2021

\begin{abstract}
In this paper, we show that the difference between the empirical estimator and the Conditional value-at-risk can be written as a simple partial sum + a residual term. Starting from this decomposition, we prove a central limit theorem and some almost sure results for the empirical estimator, for a large class of stationary sequences. We also construct a confidence interval with asymptotic level $1-\alpha$, and we study its coverage level through two different sets of simulation.
\end{abstract}

Mathematics subject classifications. (2020) - 62M10, 62G30, 60F05, 60F15

Keywords : Average value-at-risk, Conditional value-at-risk, Expected shortfall, Superquantiles, Stationary sequences, Mixing sequences

Running head : Behaviour of superquantiles/CVaR estimators

\section{Introduction}

Let $X$ be a real-valued random variable with cumulative distribution function $F$, and denote by $F^{-1}$ the generalised inverse of $F$. Assume that $\mathbb{E}(-\min (0, X))<\infty$, and let $u \in] 0,1[$. We are interested in estimating the quantity

$$
\frac{1}{u} \int_{0}^{u} F^{-1}(s) d s
$$

from the $n$ observations $X_{1}, X_{2}, \ldots, X_{n}$ of a strictly stationary and ergodic sequence $\left(X_{i}\right)_{i \in \mathbb{Z}}$ of real valued random variables, each distributed as $X$. Note that, if $F$ is continuous at $F^{-1}(u)$ then $\frac{1}{u} \int_{0}^{u} F^{-1}(s) d s=\mathbb{E}\left(X \mid X<F^{-1}(u)\right)$.

* Jérôme Dedecker, Université de Paris, CNRS, MAP5, UMR 8145, 45 rue des Saints-Pères, F-75006 Paris, France.

${ }^{\dagger}$ Florence Merlevède, LAMA, Univ Gustave Eiffel, Univ Paris Est Créteil, UMR 8050 CNRS, F-77454 Marne-La-Vallée, France. 
The natural plug in estimator of (1) is then

$$
\frac{1}{u} \int_{0}^{u} F_{n}^{-1}(s) d s
$$

where $F_{n}$ is the empirical distribution function associated with $\left(X_{1}, X_{2}, \ldots, X_{n}\right)$ and $F_{n}^{-1}$ is its generalised inverse. Note that this estimator can always be written as

$$
\frac{1}{u} \int_{0}^{u} F_{n}^{-1}(s) d s=\frac{1}{n u} \sum_{i=1}^{\lfloor n u\rfloor} X_{i, n}+\frac{(n u-\lfloor n u\rfloor)}{n u} X_{\lceil n u\rceil, n},
$$

where $\lfloor x\rfloor$ is such that $\lfloor x\rfloor \leq x<\lfloor x\rfloor+1,\lceil x\rceil$ is such that $\lceil x\rceil-1<x \leq\lceil x\rceil$, and $X_{1, n} \leq X_{2, n} \leq \cdots \leq X_{n, n}$ is the re-ordered sample built from $\left(X_{1}, X_{2}, \ldots, X_{n}\right)$.

The quantity $-\frac{1}{u} \int_{0}^{u} F^{-1}(s) d s$ has several names in the literature : according to Acerbi and Tasche [1] it is called "Expected Shortfall" or "Conditional value-at-risk" (CVaR, first introduced in [26] for the distribution of $-X)$. According to Rachev et al. [21], it is called "Average value-at-risk". According to Rockafellar and Royset [25], it is also called "Superquantile". The interest of the Conditional Value at Risk is that it is a "coherent risk measure" as defined by Artzner et al. [3] (for more details on this particular point, see also [1], [21] and [25]).

If $X_{1}, X_{2}, \ldots, X_{n}$ are independent and identically distributed (iid) random variables, an estimator of (1) (based on the minimisation of an appropriate contrast given in [20]) has been proposed in [27]. The asymptotic normality of this estimator is proved in Theorem 2 of [27] (see also [19] for a similar result in a different context). The asymptotic normality of an estimator based on the order statistic (hence very close to (3)) is also proved in [17], but under more restrictive assumptions than in [27].

In this paper, we study the asymptotic properties of the estimator (2) for a large class of strictly stationary sequence. Our results are based on the following preliminary decomposition. For any $i \geq 1$, let

$$
Y_{i}(u)=\left(F^{-1}(u)-X_{i}\right)_{+},
$$

where $a_{+}=\max (a, 0)$ denotes the positive part of a real number $a$. From Lemma 19 (and Remark 20) of the Appendix, we have:

$$
\frac{1}{u} \int_{0}^{u}\left(F_{n}^{-1}(s)-F^{-1}(s)\right) d s=-\frac{1}{n u} \sum_{i=1}^{n}\left(Y_{i}(u)-\mathbb{E}\left(Y_{i}(u)\right)\right)+R_{n}(u),
$$

where $R_{n}(u)$ is such that

$$
\left|R_{n}(u)\right| \leq \int_{L_{n}(u)}^{U_{n}(u)}\left|F_{n}(t)-F(t)\right| d t
$$

with $L_{n}(u)=\min \left(F_{n}^{-1}(u), F^{-1}(u)\right)$ and $U_{n}(u)=\max \left(F_{n}^{-1}(u), F^{-1}(u)\right)$.

As a by-product of (5), we obtain the strong consistency of the estimator (2) for any strictly stationary and ergodic sequence $\left(X_{i}\right)_{i \in \mathbb{Z}}$ such that $\mathbb{E}\left(X_{-}\right)<\infty$ where $X_{-}=$ 
$-\min (0, X)$. This follows easily from the ergodic theorem applied to the sequence $\left(Y_{i}(u)\right)_{1 \leq i \leq n}$ and from the Glivenko-Cantelli theorem for stationary and ergodic sequences (which implies that $\left\|F_{n}-F\right\|_{\infty} \rightarrow 0$ almost surely as $n \rightarrow \infty$ ). More precisely, the following proposition holds:

Proposition 1 If $\mathbb{E}\left(X_{-}\right)<\infty$, then, for any $\left.u \in\right] 0,1[$,

$$
\frac{1}{u} \int_{0}^{u} F_{n}^{-1}(s) d s \rightarrow_{n \rightarrow \infty} \frac{1}{u} \int_{0}^{u} F^{-1}(s) d s \text { a.s. }
$$

To specify the rate of convergence in this strong law of large numbers, we shall work under mixing conditions. In this paper we shall assume that $\alpha_{1, X}(k) \rightarrow_{k \rightarrow \infty} 0$, where, for any $k \geq 0$

$$
\alpha_{1, X}(k)=\sup _{t \in \mathbb{R}}\left\|\mathbb{E}\left(\mathbf{1}_{\left\{X_{k} \leq t\right\}} \mid \mathcal{F}_{0}\right)-F(t)\right\|_{1} \text { with } \mathcal{F}_{0}=\sigma\left(X_{k}, k \leq 0\right) .
$$

This coefficient, usually called the $\alpha$-dependent coefficient, is weaker than the usual strong mixing coefficient of Rosenblatt [24]. More precisely, recall that the strong mixing coefficient of Rosenblatt [24] between two $\sigma$-algebras $\mathcal{F}$ and $\mathcal{G}$ is defined by

$$
\alpha(\mathcal{F}, \mathcal{G})=\sup _{A \in \mathcal{F}, B \in \mathcal{G}}|\mathbb{P}(A \cap B)-\mathbb{P}(A) \mathbb{P}(B)| .
$$

For a strictly stationary sequence $\left(X_{i}\right)_{i \in \mathbb{Z}}$ of real valued random variables, and the $\sigma$ algebra $\mathcal{G}_{k}=\sigma\left(X_{i}, i \geq k\right)$ define then the sequence of strong mixing coefficients as follows:

$$
\begin{aligned}
\alpha(0) & =1 \text { and } \alpha(k)=2 \alpha\left(\mathcal{F}_{0}, \sigma\left(X_{k}\right)\right) \text { for } k>0 \\
\alpha_{\infty}(0) & =1 \text { and } \alpha_{\infty}(k)=2 \alpha\left(\mathcal{F}_{0}, \mathcal{G}_{k}\right) \text { for } k>0 \text { and } \mathcal{G}_{k}=\sigma\left(X_{i}, i \geq k\right) .
\end{aligned}
$$

Between these coefficients, the following relation holds: for any positive $k$,

$$
\alpha_{1, X}(k) \leq \alpha(k) \leq \alpha_{\infty}(k)
$$

The coefficient $\alpha_{\infty}(k)$ is certainly the best known, and many results have been proved under some assumptions on this coefficient, but it has strong limitations: it is not well suited to deal with linear processes with discrete innovations or non irreducible Markov chain, which is not the case of the coefficient $\alpha_{1, X}(k)$. For instance, the coefficient $\alpha_{1, X}(k)$ can be computed for many Markov chains associated with dynamical systems that fail to be strongly mixing in the sense of Rosenblatt (see for instance [10]).

The paper is organised as follows. In Section 2, we prove a central limit theorem (CLT) for our estimator under a condition expressed in terms of the coefficients $\left(\alpha_{1, X}(k)\right)_{k \geq 0}$ and of the tail distribution of $X_{-}$, and we study the optimality of this condition. In Section 3 , we give the almost sure rates of convergence of the estimator when the conditions for the CLT are not satisfied. In Section 4, we state a strong invariance principle (implying a compact law of the iterated logarithm) under a slight reinforcement of the sufficient condition for the CLT. In Section 5, we propose an estimator of the limiting variance in the CLT, and we derive a confidence interval for the conditional value at risk. All the 
proofs are given in Section 6. In Section 7 we study the asymptotic coverage level of the confidence interval built in Section 5 through two different sets of simulations: we first simulate a non-mixing Auto-Regressive process in the sense of Rosenblatt; next we simulate a strongly mixing Markov chain with a slow rate of mixing. In Appendix (Section 8) we state and prove the lemma implying the decomposition (5); we believe that this lemma is an important tool, that can also be used in different contexts.

\section{Central limit theorem}

This section deals with the central limit theorem for the estimator (2). Since the papers by Doukhan et al. [15] and Bradley [4], it is known that in such a dependent context, the optimal condition ensuring the CLT with the standard normalization mixes the decay of the coefficients $\alpha_{1, X}(k)$ and the tail distribution of the random variables.

Before stating the main result of this section, let us recall the definition of the quantile function of a random variable. For any nonnegative random variable $Z$, the quantile function $Q_{Z}$ of $Z$ is defined by $Q_{Z}(u)=\inf \{t \geq 0: \mathbb{P}(Z>t) \leq u\}$. Recall that $X_{-}=-\min (0, X)$ is the negative part of $X$.

Theorem 2 Let $u \in] 0,1\left[\right.$. Assume that $F^{-1}$ is continuous at $u$, and that

$$
\sum_{k \geq 0} \int_{0}^{\alpha_{1, X}(k)}\left(1+Q_{X_{-}}^{2}(s)\right) d s<\infty
$$

Then

$$
\sqrt{n}\left(\frac{1}{u} \int_{0}^{u} F_{n}^{-1}(s) d s-\frac{1}{u} \int_{0}^{u} F^{-1}(s) d s\right) \rightarrow^{\mathcal{D}} \mathcal{N}\left(0, \frac{\sigma^{2}(u)}{u^{2}}\right) \text { as } n \rightarrow \infty,
$$

where $\sigma^{2}(u)=\operatorname{Var}\left(Y_{0}(u)\right)+2 \sum_{k \geq 1} \operatorname{Cov}\left(Y_{0}(u), Y_{k}(u)\right)$.

Remark 3 The fact that $F^{-1}$ is continuous at $u$ is equivalent to $\operatorname{Card}\{x: F(x)=u\} \leq 1$, which is also equivalent to: one cannot find a non empty interval $I=[a, b[$ such that $F \equiv u$ on I. It is proved in Theorem 2 of [27] that this condition is in fact necessary in the iid case for the convergence to a normal distribution.

Remark 4 Let us recall some sufficient conditions for (10) to hold (see Chapter 1 in [23]):

1. If $\left\|X_{-}\right\|_{r}<\infty$ for some $\left.\left.r \in\right] 2, \infty\right]$, then (10) is satisfied provided that

$$
\sum_{k \geq 0}(k+1)^{2 /(r-2)} \alpha_{1, X}(k)<\infty .
$$

2. If $\mathbb{E}\left(X_{-}^{2} \log \left(1+X_{-}\right)\right)<\infty$, then (10) is satisfied provided that

$$
\text { there exists } \tau>0 \text { such that } \alpha_{1, X}(k)=O(\exp (-\tau k)) \text {. }
$$


Of course, if $\alpha_{1, X}(k)=0$ for $k>m$ (for instance if the variables are $m$-dependent) then (10) is satisfied as soon as $\mathbb{E}\left(X_{-}^{2}\right)<\infty$. Note that, in the iid case, this condition is less restrictive than the condition $\mathbb{E}\left(X^{2}\right)<\infty$ in Theorem 2 of [27].

As for the usual central limit theorem for the partial sums associated with dependent random variables, one can ask about the optimality of condition (10) for the validity of the conclusion of Theorem 2. Before giving our main result about the optimality of this condition, we recall the definition of the $\beta$-mixing coefficient of a stationary sequence $\left(X_{i}\right)_{i \in \mathbb{Z}}$. Let $\mathbf{X}_{k, \infty}=\left(X_{i}\right)_{i \geq k}$ and let $\mathbb{P}_{\mathbf{X}_{k, \infty} \mid \mathcal{F}_{0}}$ be a regular conditional probability of $\mathbf{X}_{k, \infty}$ given $\mathcal{F}_{0}$. Let also $B_{1}\left(\mathbb{R}^{\mathbb{N}}\right)$ be the set of $\mathcal{B}\left(\mathbb{R}^{\mathbb{N}}\right)$-measurable functions $g$ such that $\|g\|_{\infty} \leq 1$. The $\beta$-mixing coefficients of $\left(X_{i}\right)_{i \in \mathbb{Z}}$ are then defined by

$$
\beta(k)=\left\|\sup _{g \in B_{1}\left(\mathbb{R}^{\mathbb{N}}\right)} \mathbb{P}_{\mathbf{X}_{k, \infty} \mid \mathcal{F}_{0}}(g)-\mathbb{P}_{\mathbf{X}_{k, \infty}}(g)\right\|_{1} .
$$

With this definition, it is easy to see that $\alpha_{\infty}(k) \leq \beta(k)$, where $\alpha_{\infty}(k)$ is the strong mixing coefficient defined by (9).

Theorem 5 Let $a>1$ and $f$ be a continuous, increasing and negative function on ]0,1] such that

$$
\int_{0}^{1} u^{-1 / a}(f(u))^{2} d u=+\infty .
$$

Then, there exists a stationary ergodic Markov chain $\left(U_{i}\right)_{i \in \mathbb{Z}}$ of r.v.'s with uniform distribution over $[0,1]$ satisfying

$$
0<\liminf _{n \rightarrow \infty} n^{a} \alpha_{1, U}(n) \leq \limsup _{n \rightarrow \infty} n^{a} \beta(n)<\infty,
$$

and such that, for any $u \in] 0,1[$, the convergence in distribution (20) does not hold for $\left(X_{k}\right)_{k \geq 0}$ defined by $X_{k}=f\left(U_{k}\right)$.

Note that, since $f$ is continuous and increasing, $\alpha_{1, X}(n)=\alpha_{1, U}(n)$. It then follows easily from (14) that

$$
\sum_{k \geq 0} \int_{0}^{\alpha_{1, X}(k)} Q_{X_{-}}^{2}(s) d s=\infty \quad \text { if and only if (13) is satisfied. }
$$

Hence Theorem 5 shows the optimality of condition (10) for the validity of the conclusion of Theorem 2.

Now in case where $X_{-}$is bounded, (10) reduces to $\sum_{k \geq 0} \alpha_{1, X}(k)<\infty$. Next result proves the optimality of this condition to get the conclusion of Theorem 2 .

Theorem 6 There exists a stationary ergodic Markov chain $\left(X_{i}\right)_{i \geq 0}$ of r.v.'s with values in $[0,1]$ satisfying

$$
0<\liminf _{n \rightarrow \infty} n \alpha_{1, X}(n) \leq \limsup _{n \rightarrow \infty} n \alpha_{1, X}(n)<\infty,
$$


and such that, for any $u \in] 0,1[$,

$$
\frac{\sqrt{n}}{\sqrt{\log n}}\left(\frac{1}{u} \int_{0}^{u} F_{n}^{-1}(s) d s-\frac{1}{u} \int_{0}^{u} F^{-1}(s) d s\right) \rightarrow^{\mathcal{D}} \kappa(u) N \text { as } n \rightarrow \infty,
$$

where $N$ has a standard Gaussian distribution and $\kappa(u)$ is a positive constant depending on $u$.

\section{Almost sure rates of convergence}

If condition (10) is weakened we still have rates in the strong law of large numbers in the following sense:

Theorem 7 Let $u \in] 0,1[$ and $p \in] 1,2[$. Assume that

$$
\sum_{k \geq 0}(k+1)^{p-2} \int_{0}^{\alpha_{1, X}(k)}\left(1+Q_{X_{-}}^{p}(s)\right) d s<\infty .
$$

Then

$$
n^{1-1 / p}\left(\frac{1}{u} \int_{0}^{u} F_{n}^{-1}(s) d s-\frac{1}{u} \int_{0}^{u} F^{-1}(s) d s\right) \rightarrow 0 \text { a.s. as } n \rightarrow \infty .
$$

Remark 8 Note that, as Proposition 1, Theorem 7 does not require that $F^{-1}$ is continuous at $u$.

Remark 9 Let us recall some sufficient conditions for (16) to hold (see Annexe C in [23]):

1. If $\left\|X_{-}\right\|_{r}<\infty$ for some $\left.\left.r \in\right] p, \infty\right]$, then (16) is satisfied provided that

$$
\sum_{k \geq 0}(k+1)^{(p r-2 r+p) /(r-p)} \alpha_{1, X}(k)<\infty .
$$

2. If $\mathbb{E}\left(X_{-}^{p}\left(\log \left(1+X_{-}\right)\right)^{p-1}\right)<\infty$, then (16) is satisfied provided that

$$
\text { there exists } \tau>0 \text { such that } \alpha_{1, X}(k)=O(\exp (-\tau k)) \text {. }
$$

Of course, if $\alpha_{1, X}(k)=0$ for $k>m$ (for instance if the variables are $m$-dependent) then (16) is satisfied as soon as $\mathbb{E}\left(X_{-}^{p}\right)<\infty$.

In the unbounded case, as for the central limit theorem, it is possible to comment on the optimality of the condition (16). More precisely, the following result holds: 
Theorem 10 Let $p \in] 1,2[$ and $a>p-1$. Let $f$ be a continuous, increasing and negative function on $] 0,1]$ such that

$$
\int_{0}^{1} u^{-(p-1) / a}|f(u)|^{p} d u=+\infty
$$

Then, there exists a stationary ergodic Markov chain $\left(U_{i}\right)_{i \in \mathbb{Z}}$ of r.v.'s with uniform distribution over $[0,1]$ satisfying (14) and such that setting $X_{k}=f\left(U_{k}\right)$, for any $\left.u \in\right] 0,1[$, we have

$$
\limsup _{n \rightarrow \infty} n^{1-1 / p}\left|\int_{0}^{u} F_{n}^{-1}(s) d s-\int_{0}^{u} F^{-1}(s) d s\right|=+\infty \text { a.s. }
$$

Again, it follows easily from (14) and the fact that $\alpha_{1, X}(n)=\alpha_{1, U}(n)$, that

$$
\sum_{k \geq 0}(k+1)^{p-2} \int_{0}^{\alpha_{1, X}(k)} Q_{X_{-}}^{2}(s) d s=\infty \quad \text { if and only if (17) is satisfied. }
$$

Hence Theorem 10 shows the optimality of condition (16) for the validity of the conclusion of Theorem 2.

Now in case where $X_{-}$is bounded, (10) reduces to $\sum_{k \geq 0} k^{p-2} \alpha_{1, X}(k)<\infty$. Next result proves the optimality of this condition to get the conclusion of Theorem 2.

Theorem 11 Let $p \in] 1,2\left[\right.$. There exists a stationary ergodic Markov chain $\left(X_{i}\right)_{i \geq 0}$ of r.v.'s with values in $[0,1]$ satisfying

$$
0<\liminf _{n \rightarrow \infty} n^{p-1} \alpha_{1, X}(n) \leq \limsup _{n \rightarrow \infty} n^{p-1} \alpha_{1, X}(n)<\infty,
$$

and such that, for any $u \in] 0,1[$,

$$
n^{1-1 / p}\left(\frac{1}{u} \int_{0}^{u} F_{n}^{-1}(s) d s-\frac{1}{u} \int_{0}^{u} F^{-1}(s) d s\right) \rightarrow^{\mathcal{D}} \kappa(u) S \text { as } n \rightarrow \infty,
$$

where $S$ is a non degenerate p-stable distribution and $\kappa(u)$ is a positive constant depending on $u$.

\section{Strong invariance principle}

In this section, we give a strong invariance principle for

$$
\left(\frac{1}{u} \int_{0}^{u} F_{n}^{-1}(s) d s-\frac{1}{u} \int_{0}^{u} F^{-1}(s) d s\right) .
$$

This result will hold for a stronger version of the coefficient $\alpha_{1, X}(n)$, which we define now. Let $f_{z}(x)=\mathbf{1}_{z \leq x}-F(x)$. The coefficient $\alpha_{2, X}(n)$ is defined by 


$$
\alpha_{2, X}(n)=\max \left\{\alpha_{1, X}(n), \sup _{x, y \in \mathbb{R}, j \geq i \geq n}\left\|\mathbb{E}\left(f_{x}\left(X_{i}\right) f_{y}\left(X_{j}\right) \mid \mathcal{F}_{0}\right)-\mathbb{E}\left(f_{x}\left(X_{i}\right) f_{y}\left(X_{j}\right)\right)\right\|_{1}\right\}
$$

This coefficient has been introduced in [13]. For most of the usual examples, the coefficient $\alpha_{2, X}(n)$ behaves exactly as $\alpha_{1, X}(n)$ (see [13] and [10]). Moreover, is is easy to see that

$$
\alpha_{2, X}(n) \leq \alpha_{\infty}(n) \leq \beta(n),
$$

where the mixing coefficient $\alpha_{\infty}(n)$ and $\beta(n)$ are defined by (9) and (12) respectively.

Theorem 12 Let $u \in] 0,1\left[\right.$. Assume that $F^{-1}$ is continuous at $u$ and that

$$
\sum_{k \geq 0} \int_{0}^{\alpha_{2, X}(k)}\left(1+Q_{X_{-}}^{2}(s)\right) d s<\infty \quad \text { and } \quad \sum_{k \geq 0} \sqrt{\frac{\alpha_{1, X}(k)}{k+1}}<\infty .
$$

Then, enlarging the probability space if necessary, there exists a sequence $\left(Z_{i}\right)_{i \geq 0}$ of i.i.d. Gaussian random variables with mean 0 and variance $\sigma^{2}(u) / u^{2}$ such that

$$
\left(\frac{1}{u} \int_{0}^{u} F_{n}^{-1}(s) d s-\frac{1}{u} \int_{0}^{u} F^{-1}(s) d s\right)-\frac{1}{n} \sum_{i=1}^{n} Z_{i}=o\left(\sqrt{\frac{\log \log n}{n}}\right) \text { a.s. }
$$

where $\sigma^{2}(u)=\operatorname{Var}\left(Y_{0}(u)\right)+2 \sum_{k \geq 1} \operatorname{Cov}\left(Y_{0}(u), Y_{k}(u)\right)$.

Remark 13 As usual, we infer from this strong invariance principle that: the sequence

$$
\sqrt{\frac{n}{2 \log \log n}}\left(\frac{1}{u} \int_{0}^{u} F_{n}^{-1}(s) d s-\frac{1}{u} \int_{0}^{u} F^{-1}(s) d s\right)
$$

is almost surely relatively compact, with almost sure limit set $[-\sigma(u) / u, \sigma(u) / u]$.

Remark 14 If we do not assume that $F^{-1}$ is continuous at $u$ in Theorem 12, then the residual term $\sqrt{n / \log \log n} R_{n}(u)$ (where $R_{n}(u)$ satisfies (6)) does not necessarily converge to 0 almost surely. However, it is still bounded almost surely, so that there exists $C(u)<\infty$ such that

$$
\limsup _{n \rightarrow \infty} \sqrt{\frac{n}{\log \log n}}\left|\frac{1}{u} \int_{0}^{u} F_{n}^{-1}(s) d s-\frac{1}{u} \int_{0}^{u} F^{-1}(s) d s\right| \leq C(u) \text { a.s. }
$$

Remark 15 Remark 4 applies to condition (19) of Theorem 12, with $\alpha_{2, X}$ instead of $\alpha_{1, X}$, except in the bounded case (case $r=\infty$ in Item 1 of Remark 4 ). If $\left\|X_{-}\right\|_{\infty}<\infty$, then condition (19) is satisfied if

$$
\sum_{k \geq 0} \sqrt{\frac{\alpha_{2, X}(k)}{k+1}}<\infty
$$


Remark 16 If we replace $\alpha_{2, X}(k)$ by the (more restrictive) $\alpha$-mixing coefficient $\alpha_{\infty}(k)$ defined by (9), then the second condition in (19) is useless: the strong invariance principle remains true under the condition

$$
\sum_{k \geq 0} \int_{0}^{\alpha_{\infty}(k)}\left(1+Q_{X_{-}}^{2}(s)\right) d s<\infty .
$$

In that case, the residual term $R_{n}(u)$ satisfying (6) can be handled by using the arguments in [11].

\section{Confidence Interval for the CVaR}

We start by defining a suitable estimator of the limiting variance $\sigma^{2}(u)$ defined in Theorem 2 , and we prove its consistency (see Proposition 17 below). For any $i \geq 1$, let

$$
Y_{i, n}(u)=\left(F_{n}^{-1}(u)-X_{i}\right)_{+} \text {and } Y_{i, n}^{(0)}(u)=Y_{i, n}(u)-\bar{Y}_{n, n}(u),
$$

where $\bar{Y}_{n, n}(u)=n^{-1} \sum_{i=1}^{n} Y_{i, n}(u)$. Define then for any integer $k \in[0, n]$,

$$
\hat{\gamma}_{k}(u)=\frac{1}{n} \sum_{i=1}^{n-k} Y_{i, n}^{(0)}(u) Y_{i+k, n}^{(0)}(u)
$$

and

$$
\hat{c}_{n}(u)=\hat{\gamma}_{0}(u)+2 \sum_{k=1}^{v_{n}} \hat{\gamma}_{k}(u) .
$$

Proposition 17 Let $u \in[0,1]$ and $\left(v_{n}\right)_{n \geq 1}$ be a sequence of positive integers. Assume that (10) is satisfied and that

$$
v_{n} \rightarrow \infty \text { and } v_{n}^{3} \mathbb{E}\left\{X_{-}^{2}\left(1 \wedge n^{-1} X_{-}^{2}\right)\right\} \rightarrow 0 \text { as } n \rightarrow \infty .
$$

Suppose, in addition, that $F^{-1}$ is differentiable at $u$. Then

$$
\hat{c}_{n}(u) \rightarrow \sigma^{2}(u) \text { in probability, as } n \rightarrow \infty,
$$

where $\hat{c}_{n}(u)$ is defined by $(21)$.

Remark 18 Note that if $\mathbb{E}\left(X_{-}^{2}\right)<\infty$, one can always find a sequence $v_{n}$ such that (22) is satisfied. Moreover, if $\mathbb{E}\left(X_{-}^{4}\right)<\infty$, then any $v_{n}=o\left(n^{1 / 3}\right)$ satisfies (22).

When $\sigma^{2}(u)>0$, Theorem 2 combined with Proposition 17 lead to asymptotic confidence intervals for the $\mathrm{CVaR}-\frac{1}{u} \int_{0}^{u} F^{-1}(s) d s$, namely:

$$
\left[-\frac{1}{u} \int_{0}^{u} F_{n}^{-1}(s) d s-q_{1-\alpha / 2} \times \frac{\sqrt{\hat{c}_{n}(u)}}{u \sqrt{n}},-\frac{1}{u} \int_{0}^{u} F_{n}^{-1}(s) d s+q_{1-\alpha / 2} \times \frac{\sqrt{\hat{c}_{n}(u)}}{u \sqrt{n}}\right]
$$

is a confidence interval of asymptotic level $1-\alpha$ for $-\frac{1}{u} \int_{0}^{u} F^{-1}(s) d s$ (here, as usual, $q_{1-\alpha / 2}$ is the quantile of order $1-\alpha / 2$ of the standard Gaussian distribution). 


\section{Proofs}

In the proofs, for the sake of simplicity, we shall use the notation $a_{n} \ll b_{n}$, meaning that $a_{n}=O\left(b_{n}\right)$.

\subsection{Proof of Theorem 2}

We start from the decomposition (5) and we note that Theorem 1 in [14] together with ergodicity entail that

$$
\frac{1}{\sqrt{n}} \sum_{i=1}^{n}\left(Y_{i}(u)-\mathbb{E}\left(Y_{i}(u)\right)\right) \rightarrow^{\mathcal{D}} \mathcal{N}\left(0, \sigma^{2}(u)\right)
$$

provided

$$
\sum_{k \geq 0} \int_{0}^{\alpha_{1, X}(k)} Q_{Y_{0}(u)}^{2}(s) d s<+\infty .
$$

Indeed, the fact that (23) implies that $\sum_{k \geq 0} \| Y_{0}(u) \mathbb{E}\left(Y_{k}(u)-\mathbb{E}\left(Y_{k}(u) \mid \mathcal{F}_{0}\right) \|_{1}<\infty\right.$ and then their condition (1.3) can be proved by using the arguments given at the beginning of [14, Section 6] together with the fact that for any real $a, x \mapsto(a-x)_{+}$is monotone. Next, we note that, for any $u \in[0,1]$, condition $(23)$ is satisfied as soon as (10) is. Indeed it suffices to notice that for any real $a, \mathbb{P}\left((a-X)_{+}>t\right) \sim_{t \rightarrow+\infty} \mathbb{P}(\max (0,-X)>t)$. Therefore the theorem will follow if one can prove that $\sqrt{n} R_{n}(u) \rightarrow^{\mathbb{P}} 0$, as $n \rightarrow \infty$. With this aim, we recall that since it is assumed that $F^{-1}$ is continuous at $u$, we have $F_{n}^{-1}(u) \rightarrow F^{-1}(u)$ almost surely, as $n \rightarrow \infty$. Therefore, taking into account (6), to prove that $\sqrt{n} R_{n}(u) \rightarrow^{\mathbb{P}} 0$, as $n \rightarrow \infty$ it is enough to show that

$$
\lim _{\delta \rightarrow 0} \limsup _{n \rightarrow \infty} \sqrt{n} \int_{\left[F^{-1}(u)-\delta, F^{-1}(u)+\delta\right]}\left|F_{n}(t)-F(t)\right| d t=0 \text { in probability. }
$$

We shall rather prove the above convergence in $\mathbb{L}^{1}$. Note that, by $(10), \sum_{k \geq 0} \alpha_{1, X}(k)<\infty$. Therefore

$$
\left\|F_{n}(t)-F(t)\right\|_{1} \leq\left\|F_{n}(t)-F(t)\right\|_{2} \ll n^{-1 / 2} .
$$

Hence

$$
\left\|\sqrt{n} \int_{\left[F^{-1}(u)-\delta, F^{-1}(u)+\delta\right]}\left|F_{n}(t)-F(t)\right| d t\right\|_{1} \ll \delta,
$$

which proves (24). This ends the proof of the theorem.

\subsection{Proof of Theorem 5}

Let $a>1$. We consider the same stationary ergodic Markov chain $\left(U_{i}\right)_{i \in \mathbb{Z}}$ of r.v.'s with uniform distribution over $[0,1]$ as the one constructed in Theorem 5 of [15]. This chain satisfies

$$
0<\liminf _{n \rightarrow \infty} n^{a} \beta(n) \leq \limsup _{n \rightarrow \infty} n^{a} \beta(n)<\infty
$$


In addition, since (13) implies that for any $c<0, \int_{0}^{1} u^{-1 / a}(c-f(u))_{+}^{2} d u=+\infty$, Theorem 5 in [15] asserts that for $X_{k}=f\left(U_{k}\right)$ and $Y_{k}(u)=\left(F^{-1}(u)-X_{k}\right)_{+}$, the sequence $n^{-1 / 2} \sum_{k=1}^{n}\left(Y_{k}(u)-\mathbb{E}\left(Y_{k}(u)\right)\right)$ does not converge in distribution to a Gaussian random variable. Next, considering the decomposition (5), we show that, for any $u \in] 0,1[$ (since $F^{-1}$ is continuous at $\left.u\right), \sqrt{n} R_{n}(u) \rightarrow^{\mathbb{P}} 0$, as $n \rightarrow \infty$ where $R_{n}(u)$ satisfies (6). This follows by using the same arguments as those developed in the proof of Theorem 2 since (14) implies that $\sum_{k \geq 1} \beta(k)<\infty$ and then $\sum_{k \geq 1} \alpha_{1, X}(k)<\infty$. So, to sum up, we have proved that it is possible to construct a stationary ergodic Markov chain $\left(U_{i}\right)_{i \in \mathbb{Z}}$ of r.v.'s with uniform distribution over $[0,1]$ whose $\beta$-mixing coefficients satisfy (25), satisfying $\sum_{k \geq 0} \int_{0}^{\beta(k)} Q_{X_{-}}^{2}(s) d s=\infty$ where $X=f(U)$ with $f$ a continuous, increasing and negative function on $] 0,1]$, and such that for any $u \in] 0,1[$,

$$
\sqrt{n}\left(\int_{0}^{u} F_{n}^{-1}(s) d s-\int_{0}^{u} F^{-1}(s) d s\right) \text { does not converge in distribution. }
$$

To end the proof, it remains to show that (14) is fully satisfied, or equivalently (since $f$ is continuous and increasing) that $\liminf _{n \rightarrow \infty} n^{a} \alpha_{1, X}(n)>0$. This can be done using the same arguments as those developed in the first part of the proof of [15, Corollary 1], namely, in the proof of (4.22).

\subsection{Proof of Theorem 6}

Let $\gamma \in] 0,1\left[\right.$. We consider the Markov chain $\left(X_{k}\right)_{k \geq 1}$ associated with the transformation $T_{\gamma}$ defined from $[0,1]$ to $[0,1]$ by

$$
T_{\gamma}(x)= \begin{cases}x\left(1+2^{\gamma} x^{\gamma}\right) & \text { if } x \in[0,1 / 2[ \\ 2 x-1 & \text { if } x \in[1 / 2,1]\end{cases}
$$

This is the so-called LSV [18] map with parameter $\gamma$. There exists a unique $T_{\gamma}$-invariant measure $\nu_{\gamma}$ on $[0,1]$, which is absolutely continuous with respect to the Lebesgue measure with positive density denoted by $h_{\gamma}$. We denote by $K_{\gamma}$ the Perron-Frobenius operator of $T_{\gamma}$ with respect to $\nu_{\gamma}$. Recall that for any bounded measurable functions $f$ and $g$, $\nu_{\gamma}\left(f \cdot g \circ T_{\gamma}\right)=\nu_{\gamma}\left(K_{\gamma}(f) g\right)$. Let then $\left(X_{i}\right)_{i \geq 0}$ be the stationary Markov chain with transition Kernel $K_{\gamma}$ and invariant measure $\nu_{\gamma}$. Since $\nu_{\gamma}$ has a positive density $h_{\gamma}$ on ]0, 1[, it follows that $F^{-1}$ is continuous on $] 0,1[$.

We take now $\gamma=1 / 2$. From Section 1.4 in [10], we know that (15) holds. Next, we follow the lines of the proof of Theorem 2 with the following modifications. From Comment 2 page 88 in Gouezel [16], since $g_{u}: x \mapsto\left(F^{-1}(u)-x\right)_{+}$is Lipshitz and $k(u):=g_{u}(0)-$ $\int_{0}^{1} g_{u}(x) h_{1 / 2}(x) d x>0$, we derive

$$
\frac{1}{\sqrt{n \log n}} \sum_{i=1}^{n}\left(g_{u}\left(X_{i}\right)-\mathbb{E}\left(g_{u}\left(X_{i}\right)\right)\right) \rightarrow^{\mathcal{D}} \sqrt{h_{1 / 2}(1 / 2)} k(u) N,
$$


where $N$ has a standard Gaussian distribution. On another hand,

$$
\left\|F_{n}(t)-F(t)\right\|_{2}^{2} \ll \sum_{k=0}^{n} \alpha_{1, X}(k) \ll \frac{\log (n)}{n},
$$

which implies that $\sqrt{\frac{n}{\log n}} R_{n}(u) \rightarrow^{\mathbb{P}} 0$ where $R_{n}(u)$ satisfies (6). The result then follows from the decomposition (5).

\subsection{Proof of Theorem 7}

We start from the decomposition (5) and we note that Corollary 3.2 in [23] implies that, almost surely,

$$
\frac{1}{n^{1 / p}} \sum_{i=1}^{n}\left(Y_{i}(u)-\mathbb{E}\left(Y_{i}(u)\right)\right) \rightarrow 0, \text { as } n \rightarrow \infty
$$

provided

$$
\sum_{k \geq 0}(k+1)^{p-2} \int_{0}^{\alpha_{1, X}(k)} Q_{Y_{0}(u)}^{p}(s) d s<\infty .
$$

Next, condition (26) clearly holds provided that (16) is satisfied.

It remains to prove that, almost surely,

$$
n^{(p-1) / p} \int_{\left[L_{n}(u), U_{n}(u)\right]}\left|F_{n}(t)-F(t)\right| d t \rightarrow 0, \text { as } n \rightarrow \infty .
$$

With this aim, note that by (35), almost surely, there exists an integer $N$ such that for any $n \geq N$,

$$
a:=F^{-1}(u / 2) \leq F_{n}^{-1}(u) \leq F^{-1}((1+u) / 2):=b .
$$

Hence, to prove (27) it is enough to prove that

$$
n^{(p-1) / p} \int_{a}^{b}\left|F_{n}(t)-F(t)\right| d t \rightarrow 0, \text { as } n \rightarrow \infty .
$$

Since $\sum_{k \geq 0}(k+1)^{p-2} \alpha_{1, X}(k)<\infty$, this follows from the proof of Proposition 3.3 in [5] by considering that the random variables $X_{k}$ are bounded. This ends the proof of the theorem.

\subsection{Proof of Theorem 10}

Let $a>p-1$.We consider the same stationary ergodic Markov chain $\left(U_{i}\right)_{i \in \mathbb{Z}}$ of r.v.'s with uniform distribution over $[0,1]$ as in the proof of Theorem 5. This chain satisfies (25). In 
addition, since (17) implies that for any $c<0, \int_{0}^{1} u^{-(p-1) / a}(c-f(u))_{+}^{p} d u=+\infty$, Theorem 2 in [22] asserts that for $X_{k}=f\left(U_{k}\right)$ and $Y_{k}(u)=\left(F^{-1}(u)-X_{k}\right)_{+}$,

$$
\limsup _{n \rightarrow \infty} \frac{1}{n^{1 / p}}\left|\sum_{k=1}^{n}\left(Y_{k}(u)-\mathbb{E}\left(Y_{k}(u)\right)\right)\right|=\infty \quad \text { a.s. }
$$

Next, considering the decomposition (5), we show that, for any $u \in] 0,1\left[, n^{(p-1) / p} R_{n}(u) \rightarrow\right.$ 0 a.s., as $n \rightarrow \infty$ where $R_{n}(u)$ satisfies (6). The proof is exactly the same as the proof of (27).

So, to sum up, we have proved that it is possible to construct a stationary ergodic Markov chain $\left(U_{i}\right)_{i \in \mathbb{Z}}$ of r.v.'s with uniform distribution over $[0,1]$ whose $\beta$-mixing coefficients satisfy (25), satisfying $\sum_{k \geq 0}(k+1)^{p-2} \int_{0}^{\beta(k)} Q_{X_{-}}^{p}(s) d s=\infty$ where $X=f(U)$ with $f$ a continuous, increasing and negative function on $] 0,1]$, and such that for any $u \in] 0,1[$,

$$
\limsup _{n \rightarrow \infty} n^{(p-1) / p}\left|\int_{0}^{u} F_{n}^{-1}(s) d s-\int_{0}^{u} F^{-1}(s) d s\right|=\infty .
$$

The fact that (14) is fully satisfied is proved as in the proof of Theorem 5 .

\subsection{Proof of Theorem 11}

We consider the Markov chain $\left(X_{k}\right)_{k \geq 1}$ described in the proof of Theorem 6 with $\gamma=1 / p$. From Section 1.4 in [10], we know that (18) holds. From Theorem 1.3 in Gouezel [16], since $g_{u}: x \mapsto\left(F^{-1}(u)-x\right)_{+}$is Lipshitz and $k(u):=g_{u}(0)-\int_{0}^{1} g_{u}(x) h_{1 / p}(x) d x>0$, we derive

$$
\frac{1}{n^{1 / p}} \sum_{i=1}^{n}\left(g_{u}\left(X_{i}\right)-\mathbb{E}\left(g_{u}\left(X_{i}\right)\right)\right) \rightarrow^{\mathcal{D}} C_{p} k(u) S
$$

where $S$ is a non-degenerate $p$-stable distribution and $C_{p}>0$. On another hand, we know from Theorem 1.1 in [9] that

$$
n^{1-1 / p}\left(\int_{0}^{1}\left(F_{n}(t)-F(t)\right)^{2} d t\right)^{1 / 2} \text { converges in distribution as } n \rightarrow \infty .
$$

Now, the quantity $R_{n}(u)$ defined by (6) satisfies

$$
n^{1-1 / p} R_{n}(u) \leq n^{1-1 / p}\left(\int_{0}^{1}\left(F_{n}(t)-F(t)\right)^{2} d t\right)^{1 / 2} \sqrt{\left|F_{n}^{-1}(u)-F^{-1}(u)\right|} .
$$

Since $F^{-1}$ is continuous at $u, F_{n}^{-1}(u)$ converges almost surely to $F^{-1}(u)$, which implies that $n^{1-1 / p} R_{n}(u) \rightarrow^{\mathbb{P}} 0$. The result follows from the decomposition (5). 


\subsection{Proof of Theorem 12}

We start from the decomposition (5), and we note that Theorem 1.13 in [10] implies that: there exists a sequence $\left(Z_{i}\right)_{i \geq 0}$ of i.i.d. Gaussian random variables with mean 0 and variance $\sigma^{2}(u) / u$ such that

$$
\frac{1}{n u} \sum_{i=1}^{n}\left(Y_{i}(u)-\mathbb{E}\left(Y_{i}(u)\right)\right)-\frac{1}{n} \sum_{i=1}^{n} Z_{i}=o\left(\sqrt{\frac{\log \log n}{n}}\right) \text { a.s. }
$$

provided

$$
\sum_{k \geq 0} \int_{0}^{\alpha_{2, X}(k)} Q_{Y_{0}(u)}^{2}(s) d s<+\infty
$$

It remains to prove that

$$
\lim _{n \rightarrow \infty} \sqrt{\frac{n}{\log \log n}} R_{n}(u)=0 \text { a.s. }
$$

where $R_{n}(u)$ satisfies $(6)$. Since $F^{-1}$ is continuous at $u, F_{n}^{-1}(u)$ converges to $F^{-1}$ almost surely. Hence (30) follows from

$$
\lim _{\delta \rightarrow 0} \limsup _{n \rightarrow \infty} \sqrt{\frac{n}{\log \log n}} \int_{F^{-1}(u)-\delta}^{F^{-1}(u)+\delta}\left|F_{n}(t)-F(t)\right| d t=0 \text { a.s. }
$$

Applying Theorem 1.1 in [7] to the space $\mathbb{L}_{1}\left(\left[F^{-1}(u)-\delta, F^{-1}(u)+\delta\right], d t\right)$, we get that

$$
\limsup _{n \rightarrow \infty} \sqrt{\frac{n}{\log \log n}} \int_{F^{-1}(u)-\delta}^{F^{-1}(u)+\delta}\left|F_{n}(t)-F(t)\right| d t \leq 2 C \delta \sum_{k \geq 0} \sqrt{\frac{\alpha_{1, X}(k)}{k+1}} \text { a.s. }
$$

for some universal constant $C$, and (31) easily follows.

\subsection{Proof of Proposition 17}

Recall the notation (4), namely: for any $i \geq 1, Y_{i}(u)=\left(F^{-1}(u)-X_{i}\right)_{+}$. For any integer $k \in[0, n]$, let

$$
\gamma_{k}^{*}(u)=\frac{1}{n} \sum_{i=1}^{n-k}\left(Y_{i}(u)-\bar{Y}_{n}(u)\right)\left(Y_{i+k}(u)-\bar{Y}_{n}(u)\right)
$$

and, for any sequence of positive integers $\left(v_{n}\right)_{n \geq 1}$ define

$$
c_{n}(u)=\gamma_{0}^{*}(u)+2 \sum_{k=1}^{v_{n}} \gamma_{k}^{*}(u) .
$$

According to $[8$, Theorem $1(\mathrm{~b})]$, if (10) is satisfied and if

$$
v_{n} \rightarrow \infty \text { and } v_{n}^{3} \mathbb{E}\left\{Y_{0}^{2}(u)\left(1 \wedge n^{-1} Y_{0}^{2}(u)\right)\right\} \rightarrow 0 \text { as } n \rightarrow \infty,
$$


then

$$
c_{n}(u) \rightarrow \sigma^{2}(u) \text { in probability, as } n \rightarrow \infty .
$$

Note that condition (32) holds as soon as condition (22) does. Next, for any positive integer $i$, noticing that $\left|Y_{i}(u)-Y_{i, n}(u)\right| \leq\left|F_{n}^{-1}(u)-F^{-1}(u)\right|$, we infer that

$$
\begin{aligned}
& \left|\left(Y_{i}(u)-\bar{Y}_{n}(u)\right)\left(Y_{i+k}(u)-\bar{Y}_{n}(u)\right)-Y_{i, n}^{(0)}(u) Y_{i+k, n}^{(0)}(u)\right| \\
& \quad \leq 2\left|F_{n}^{-1}(u)-F^{-1}(u)\right|\left(\left|Y_{i}(u)\right|+\bar{Y}_{n}(u)\right) \\
& +2\left|F_{n}^{-1}(u)-F^{-1}(u)\right|\left(\left|Y_{i+k}(u)\right|+\bar{Y}_{n}(u)\right)+4\left|F_{n}^{-1}(u)-F^{-1}(u)\right|^{2} .
\end{aligned}
$$

Therefore,

$$
\left|c_{n}(u)-\hat{c}_{n}(u)\right| \leq 8 v_{n}\left|F_{n}^{-1}(u)-F^{-1}(u)\right| \times \frac{1}{n} \sum_{i=1}^{n}\left|Y_{i}(u)\right|+4 v_{n}\left|F_{n}^{-1}(u)-F^{-1}(u)\right|^{2} .
$$

But, by the ergodic theorem, $n^{-1} \sum_{i=1}^{n}\left|Y_{i}(u)\right| \rightarrow \mathbb{E}\left(\left|Y_{1}(u)\right|\right)$ a.s., as $n \rightarrow \infty$. Therefore, taking into account (33), the proposition will follow if one can prove that

$$
v_{n}\left|F_{n}^{-1}(u)-F^{-1}(u)\right| \rightarrow 0 \text { in probability, as } n \rightarrow \infty .
$$

With this aim, we start with the well-known inequalities:

$$
F^{-1}\left(u-\epsilon_{n, 1}\right) \leq F_{n}^{-1}(u) \leq F^{-1}\left(u+\epsilon_{n, 2}\right),
$$

where $\epsilon_{n, 1}=\left\|F_{n}-F\right\|_{\infty} \wedge u$ and $\epsilon_{n, 2}=\left\|F_{n}-F\right\|_{\infty} \wedge(1-u)$. Next, since $F^{-1}$ is differentiable at $u$, we have

$$
F^{-1}\left(u-\epsilon_{n, 1}\right)-F^{-1}(u)=-\left(F^{-1}\right)^{\prime}(u) \times \epsilon_{n, 1}+o\left(\left\|F_{n}-F\right\|_{\infty}\right) .
$$

Similarly,

$$
F^{-1}\left(u+\epsilon_{n, 2}\right)-F^{-1}(u)=\left(F^{-1}\right)^{\prime}(u) \times \epsilon_{n, 2}+o\left(\left\|F_{n}-F\right\|_{\infty}\right) .
$$

So, overall, since $F^{-1}$ is assumed to be differentiable at $u$, to prove (34) it is enough to prove that

$$
v_{n}\left\|F_{n}-F\right\|_{\infty} \rightarrow 0 \text { in probability, as } n \rightarrow \infty .
$$

For any fixed positive integer $p$, setting $t_{i}=F^{-1}(p)$ for any integer $i \in[1, p-1], t_{0}=-\infty$ and $t_{p}=+\infty$, and defining

$$
V_{n}=\max _{0 \leq i \leq p-1}\left(\left|F_{n}\left(t_{i}\right)-F\left(t_{i}\right)\right|,\left|F_{n}\left(t_{i+1}^{-}\right)-F\left(t_{i+1}^{-}\right)\right|\right):=\max _{0 \leq i \leq p-1} Z_{i, n},
$$

we have, for any $t \in \mathbb{R}$,

$$
\left|F_{n}(t)-F(t)\right| \leq V_{n}+\frac{1}{p}
$$

But

$$
\left\|V_{n}\right\|_{2} \leq \sqrt{p} \max _{1 \leq i \leq p}\left\|Z_{i, n}\right\|_{2} .
$$


Since $\sum_{k \geq 1} \alpha_{1, X}(k)<\infty,\left\|Z_{i, n}\right\|_{2} \ll n^{-1 / 2}$, we get

$$
\left\|\sup _{t \in \mathbb{R}}\left|F_{n}(t)-F(t)\right|\right\|_{2} \ll \sqrt{\frac{p}{n}}+\frac{1}{p} \ll n^{-1 / 3},
$$

by choosing $p=\left[n^{1 / 3}\right]$. Since by assumption $v_{n}=o\left(n^{1 / 3}\right)$, (36) follows. This ends the proof of the proposition.

\section{Simulations}

In this section, we shall simulate two different stationary processes, in order to estimate the coverage level of the confidence interval presented in Section 5. The first process is non mixing in the sense of Rosenblatt [24], but its coefficients $\alpha_{1, X}(k)$ decrease at an exponential rate. The second process is a $\beta$-mixing process (hence mixing in the sense of Rosenblatt), with a slow rate of mixing.

\subsection{Example 1: Non mixing auto-regressive model}

We first simulate $\left(Y_{1}, \ldots, Y_{n}\right)$, according to the simple $\operatorname{AR}(1)$ equation

$$
\text { for } k \geq 1, \quad Y_{k+1}=\frac{1}{2}\left(Y_{k}+\varepsilon_{k+1}\right)
$$

where $Y_{1}$ is uniformly distributed over $[0,1]$, and $\left(\varepsilon_{i}\right)_{i \geq 2}$ is a sequence of iid random variables with distribution $\mathcal{B}(1 / 2)$, independent of $Y_{1}$.

One can check that the transition Kernel of the chain $\left(Y_{i}\right)_{i \geq 1}$ is

$$
K(f)(x)=\frac{1}{2}\left(f\left(\frac{x}{2}\right)+f\left(\frac{x+1}{2}\right)\right),
$$

and that the uniform distribution on $[0,1]$ is the unique invariant distribution by $K$. Hence, the chain $\left(Y_{i}\right)_{i \geq 1}$ is strictly stationary.

It is well known that the chain $\left(Y_{i}\right)_{i \geq 1}$ is not $\alpha$-mixing in the sense of Rosenblatt [24] (see for instance [2]). However, one can prove that the coefficients $\alpha_{1, Y}$ of $\left(Y_{i}\right)_{i \geq 1}$ are such that

$$
\alpha_{1, Y}(k) \leq 2^{-k}
$$

(see for instance Section 6.1 in [13]). Note that this upper bound is also valid for the coefficient $\alpha_{2, Y}(k)$ defined in Section 4 .

Let now $Q_{\mu, \sigma^{2}}$ be the inverse of the cumulative distribution function of the law $\mathcal{N}\left(\mu, \sigma^{2}\right)$. Let then

$$
X_{i}=Q_{\mu, \sigma^{2}}\left(Y_{i}\right)
$$

The sequence $\left(X_{i}\right)_{i \geq 1}$ is also a stationary Markov chain (as an invertible function of a stationary Markov chain), and one can easily check that $\alpha_{1, X}(k)=\alpha_{1, Y}(k)$. By construction, $X_{i}$ is $\mathcal{N}\left(\mu, \sigma^{2}\right)$-distributed. 
For $u \in] 0,1[$, we can now compute the CVaR (see for instance [21]):

$$
-\frac{1}{u} \int_{0}^{u} F^{-1}(s) d s=\frac{\sigma}{u \sqrt{2 \pi}} \exp \left(-\frac{\left(\Phi^{-1}(u)\right)^{2}}{2}\right)-\mu
$$

where $\Phi$ is the cdf of the $\mathcal{N}(0,1)$-distribution.

Now, according to Section 5,

$$
\left[-\frac{1}{u} \int_{0}^{u} F_{n}^{-1}(s) d s-q_{1-\alpha / 2} \times \frac{\sqrt{\hat{c}_{n}(u)}}{u \sqrt{n}},-\frac{1}{u} \int_{0}^{u} F_{n}^{-1}(s) d s+q_{1-\alpha / 2} \times \frac{\sqrt{\hat{c}_{n}(u)}}{u \sqrt{n}}\right]
$$

is a confidence interval of asymptotic level $1-\alpha$ for $-u^{-1} \int_{0}^{u} F^{-1}(s) d s$. Recall that $\hat{c}_{n}(u)$ is the estimator of $\sigma^{2}(u)$ built in Proposition 17 (for some $v_{n}$ such that $v_{n}=o\left(n^{1 / 3}\right)$ ).

In the simulations, we shall take $u=0.75, \alpha=0.05, \mu=1, \sigma^{2}=1$, so that $X_{i}$ is $\mathcal{N}(1,1)$-distributed. We shall estimate the variance of $\sqrt{n} \int_{0}^{u} F_{n}^{-1}(s) d s / \sqrt{\hat{c}_{n}(u)}$ and the coverage probability of the interval (38) via a basic Monte Carlo procedure over $N=2000$ independent trials, for different values of $n$, with the choice $v_{n}=\left[n^{1 / 4}\right]$. This will give us Var $_{1}$ and Cove $_{1}$.

We shall also try another computation for $\hat{c}_{n}(u)$. We shall fit an autoregressive process (with an automatic procedure based on the AIC criterion) on the "residuals" $Y_{i, n}^{(0}(u)$ defined in Section 5, and then compute $\hat{c}_{n}(u)$ as the covariance series of the fitted AR process (this procedure is inspired from the paper [6], where it is shown to work well in the context of linear regression). This will then gives us $\operatorname{Var}_{2}$ and $\mathrm{Cove}_{2}$.

The results are presented in the following table.

\begin{tabular}{|c|c|c|c|c|c|c|c|c|c|}
\hline$n$ & 200 & 300 & 400 & 500 & 600 & 700 & 800 & 900 & 1000 \\
\hline Var $_{1}$ & 1.24 & 1.201 & 1.12 & 1.091 & 1.111 & 1.053 & 1.113 & 1.08 & 1.041 \\
\hline Var $_{2}$ & 1.153 & 1.143 & 1.08 & 1.041 & 1.067 & 1.021 & 1.08 & 1.043 & 1.02 \\
\hline Cove $_{1}$ & 0.927 & 0.931 & 0.937 & 0.938 & 0.939 & 0.94 & 0.937 & 0.94 & 0.944 \\
\hline Cove $_{2}$ & 0.933 & 0.935 & 0.941 & 0.946 & 0.942 & 0.943 & 0.941 & 0.942 & 0.946 \\
\hline
\end{tabular}

We see that both procedures work well: the coverage levels are greater than $93 \%$ for $n \geq 300$ and very close to $95 \%$ for $n=1000$. We see that the second procedure (with and automatic AR fitting on the residuals) is always slightly better (better coverage and variance closer to 1 ).

\subsection{Example 2: $\beta$-mixing Markov chain}

In this section, we first simulate the stationary $\beta$-mixing Markov chain introduced in [15] as follows. Let $a>1$, and let $\nu$ and $\pi$ be the two probability measures on $[0,1]$ with respective densities $f_{\nu}(x)=a x^{a-1} \mathbf{1}_{0 \leq x \leq 1}$ and $f_{\pi}(x)=(a+1) x^{a} \mathbf{1}_{0 \leq x \leq 1}$. Let $F_{\pi}$ be the cdf of $\pi$, and let $Y_{1}$ be a random variable with law $\nu$. Let $\left(\varepsilon_{i}\right)_{i \geq 1}=\left(\left(U_{i}, V_{i}\right)\right)_{i \geq 2}$ be a sequence of iid random variables with uniform law over $[0,1]^{2}$ and independent of $Y_{1}$. For $k \geq 1$ define then

$$
Y_{k+1}=Y_{k} \mathbf{1}_{U_{k+1} \geq Y_{k}}+F_{\pi}^{-1}\left(V_{k+1}\right) \mathbf{1}_{U_{k+1}<Y_{k}}
$$


One can check that this chain is strictly stationary, with invariant distribution $\nu$. Moreover, it is proved in [15] that the $\beta$-mixing coefficients of this chain are exactly of order $n^{-a}$. Note also that $Y_{i}^{a}$ is uniformly distributed over $[0,1]$.

Let then

$$
X_{i}=Q_{\mu, \sigma^{2}}\left(Y_{i}^{a}\right) .
$$

The sequence $\left(X_{i}\right)_{i \geq 1}$ is also a stationary Markov chain (as an invertible function of a stationary Markov chain), and its $\beta$-mixing coefficients are such that: there exist $B>$ $A>0$ such that, for any $n \geq 1, A n^{-a} \leq \beta(n) \leq B n^{-a}$. By construction $X_{i}$ is $\mathcal{N}\left(\mu, \sigma^{2}\right)$ distributed.

Note that the equality (37) is still valid, and that (38) is a confidence interval of asymptotic level $1-\alpha$ for $-u^{-1} \int_{0}^{u} F^{-1}(s) d s$.

In the simulations, we shall take $u=0.75, \alpha=0.05, \mu=1, \sigma^{2}=1$, so that $X_{i}$ is $\mathcal{N}(1,1)$ distributed. We choose $a=3$, which gives a rather slow rate of mixing. We then proceed exactly as in Subsection 7.1 to estimate the quantities $\operatorname{Var}_{1}, \operatorname{Cove}_{1}\left(\right.$ with $v_{n}=\left[n^{1 / 4}\right]$ ), $\operatorname{Var}_{2}$ and Cove $_{2}$.

The results are presented in the following table.

\begin{tabular}{|r|c|c|c|c|c|c|c|c|c|}
\hline$n$ & 300 & 400 & 500 & 600 & 700 & 800 & 900 & 1000 & 4000 \\
\hline $\operatorname{Var}_{1}$ & 1.42 & 1.41 & 1.26 & 1.326 & 1.293 & 1.283 & 1.261 & 1.242 & 1.161 \\
\hline Var $_{2}$ & 1.314 & 1.289 & 1.146 & 1.18 & 1.201 & 1.208 & 1.177 & 1.14 & 1.093 \\
\hline Cove $_{1}$ & 0.899 & 0.902 & 0.914 & 0.912 & 0.915 & 0.921 & 0.915 & 0.923 & 0.935 \\
\hline Cove $_{2}$ & 0.905 & 0.912 & 0.924 & 0.928 & 0.925 & 0.93 & 0.93 & 0.94 & 0.944 \\
\hline
\end{tabular}

Since the rate of mixing is quite slow, the results are less satisfactory than for the first example. One has to take $n \geq 800$ to get a coverage greater than $93 \%$ for the second method (with an automatic AR fitting on the residuals), and $n \geq 1000$ to get a coverage greater than $92 \%$ for the method presented in Section 5 (with $v_{n}=\left[n^{1 / 4}\right]$ ). For $n=4000$, the coverage is around $93.5 \%$ for the first method and around $94.5 \%$ for the second method. As for Example 1, we see that the second procedure is always slightly better (better coverage and variance closer to 1 ).

\section{Appendix}

Lemma 19 Let $u \in] 0,1[$, and let $F$ and $G$ be two cumulative distribution functions such that $\int_{-\infty}^{0} G(t) d t<\infty$ and $\int_{-\infty}^{0} F(t) d t<\infty$. The following equalities hold:

$$
\int_{0}^{u} G^{-1}(s) d s-\int_{0}^{u} F^{-1}(s) d s=\int_{\mathbb{R}}((F(t) \wedge u)-(G(t) \wedge u)) d t
$$

and

$$
\int_{0}^{u} G^{-1}(s) d s-\int_{0}^{u} F^{-1}(s) d s=\int_{-\infty}^{F^{-1}(u)}(F(t)-G(t)) d t+R
$$


where

$$
|R| \leq \int_{[L(u), U(u)]}|F(t)-G(t)| d t .
$$

with $L(u)=\min \left(F^{-1}(u), G^{-1}(u)\right)$ and $U(u)=\max \left(F^{-1}(u), G^{-1}(u)\right)$.

Remark 20 Note that the first term on right hand in (39) satisfies

$$
\int_{-\infty}^{F^{-1}(u)}(F(t)-G(t)) d t=\mathbb{E}\left(\left(F^{-1}(u)-X\right)_{+}\right)-\mathbb{E}\left(\left(F^{-1}(u)-Y\right)_{+}\right),
$$

where $X$ and $Y$ are two random variables with cumulative distribution function $F$ and $G$ respectively.

Proof. We start by writing

$$
\int_{0}^{u} F^{-1}(s) d s=-\int_{-\infty}^{F^{-1}(u)} F(t) d t+u F^{-1}(u) .
$$

It follows that

$$
\begin{array}{r}
\int_{0}^{u} G^{-1}(s) d s-\int_{0}^{u} F^{-1}(s) d s=-\int_{-\infty}^{G^{-1}(u)} G(t) d t \\
+\int_{-\infty}^{F^{-1}(u)} F(t) d t+u\left(G^{-1}(u)-F^{-1}(u)\right) \\
=\int_{\mathbb{R}}((F(t) \wedge u)-(G(t) \wedge u)) d t \quad(40)
\end{array}
$$

and the first equality is proved. Next, from the first equality in (40), we also deduce that

$$
\int_{0}^{u} G^{-1}(s) d s-\int_{0}^{u} F^{-1}(s) d s=\int_{-\infty}^{F^{-1}(u)}(F(t)-G(t) d t+R,
$$

where

$$
R=u\left(G^{-1}(u)-F^{-1}(u)\right)-\int_{-\infty}^{G^{-1}(u)} G(t) d t+\int_{-\infty}^{F^{-1}(u)} G(t) d t .
$$

Assume that $G^{-1}(u) \leq F^{-1}(u)$. Then, since $G^{-1}(u) \leq t<F^{-1}(u) \Longleftrightarrow F(t)<u \leq G(t)$, we get

$$
|R|=\left|\int_{G^{-1}(u)}^{F^{-1}(u)}(G(t)-u) d t\right| \leq \int_{G^{-1}(u)}^{F^{-1}(u)}(G(t)-F(t)) d t .
$$

On another hand, if $F^{-1}(u) \leq G^{-1}(u)$. Then, since $F^{-1}(u) \leq t<G^{-1}(u) \Longleftrightarrow G(t)<$ $u \leq F(t)$, we get

$$
|R|=\left|\int_{F^{-1}(u)}^{G^{-1}(u)}(u-G(t)) d t\right| \leq \int_{F^{-1}(u)}^{G^{-1}(u)}(F(t)-G(t)) d t,
$$

and the Lemma is proved. 


\section{References}

[1] Acerbi, C. and Tasche D., On the coherence of expected shortfall. Journal of Banking and Finance 26 (2002) 1487-1503.

[2] Andrews, D. W. K. Nonstrong mixing autoregressive processes. J. Appl. Probab. 21 (1984), no. 4, 930-934.

[3] Artzner, P., Delbaen, F., Eber J.-M. and Heath, D. Coherent measure of Risk. Mathematical Finance 9 (1999), no 3, 203-227.

[4] Bradley, R. C. On quantiles and the central limit question for strongly mixing sequences. Dedicated to Murray Rosenblatt. J. Theoret. Probab. 10 (1997), no. 2, $507-555$.

[5] Berthet, P., Dedecker, J. and Merlevède, F. Central limit theorem and almost sure results for bivariate empirical $W_{1}$ distances. Pub. Inst. Stat. Univ. Paris 63 (2019), fasc. 2-3, 205-220.

[6] Caron, E., Dedecker, J. and Michel, B. Linear regression with stationary errors: the R package slm. https://hal.archives-ouvertes.fr/hal-02157155v2/document

[7] Cuny, C. Invariance principles under the Maxwell-Woodroofe condition in Banach spaces. Ann. Probab. 45 (2017) 1578-1611.

[8] Dedecker, J. A central limit theorem for stationary random fields. Probab. Theory Related Fields 110 (1998), no. 3, 397-426.

[9] Dedecker, J., Dehling, H., and Taqqu, M. S. Weak convergence of the empirical process of intermittent maps in $\mathbb{L}^{2}$ under long-range dependence. Stoch. Dyn. 15 (2015), no. 2, 29 pp.

[10] Dedecker, J., Gouëzel, S. and Merlevède, F. Some almost sure results for unbounded functions of intermittent maps and their associated Markov chains. Ann. Inst. Henri Poincaré Probab. Stat. 46 (2010), no. 3, 796-821.

[11] Dedecker, J. and Merlevède, F. Almost sure invariance principle for the Kantorovich distance between the empirical and the marginal distributions of strong mixing sequences. Statist. Probab. Lett. 171 (2021).

[12] Dedecker, J. and Merlevède, F. Behavior of the Wasserstein distance between the empirical and the marginal distributions of stationary $\alpha$ - dependent sequences. Bernoulli 23 (2017), no. 3, 2083-2127.

[13] Dedecker, J. and Prieur, C. An empirical central limit theorem for dependent sequences. Stochastic Process. Appl. 117 (2007), no. 1, 121-142. 
[14] Dedecker, J. and Rio, E. On the functional central limit theorem for stationary processes. Ann. Inst. H. Poincaré Probab. Statist. 36 (2000), no. 1, 1-34.

[15] Doukhan, P., Massart, P. and Rio, E. The functional central limit theorem for strongly mixing processes. Ann. Inst. H. Poincaré Probab. Statist. 30 (1994), no. 1, $63-82$.

[16] Gouëzel, S. Central limit theorem and stable laws for intermittent maps. Probab. Theory Related Fields 128 (2004), no. 1, 82-122.

[17] Labopin-Richard, T., Gamboa, F., Garivier, A., and Iooss, B. Bregman superquantiles. Estimation methods and applications. Depend. Model. 4 (2016), no. 1, 76-108.

[18] Liverani, C. Saussol, B. and Vaienti, S. A probabilistic approach to intermittency. Ergodic Theory Dynam. Systems 19 (1999), no. 3, 671-685.

[19] Nagaraja, H.. Some nondegenerate limit laws for the selection differential. The Annals of Statistics 10 (1982), no. 4, 1306-1310.

[20] Pflug, G. Some remarks on the value-at-risk and the conditional value-at-risk. In: Uryasev, S. (Ed.), Probabilistic Constrained Optimization: Methodology and Applications. (2000) Kluwer, Dordrecht.

[21] Rachev S. T., Stoyanov S., and Fabozzi F. J. Advanced Stochastic Models, Risk Assessment, and Portfolio Optimization: The Ideal Risk, Uncertainty, and Performance Measures. John Wiley, Finance, 2007

[22] Rio, E. A maximal inequality and dependent Marcinkiewicz-Zygmund strong laws. Ann. Probab. 23 (1995), no. 2, 918-937.

[23] Rio, E. Asymptotic theory of weakly dependent random processes. Translated from the 2000 French edition. Probability Theory and Stochastic Modelling, 80. Springer, Berlin, 2017. xviii+204 pp.

[24] Rosenblatt, M. A central limit theorem and a strong mixing condition. Proc. Natl. Acad. Set. USA 42 (1956), 43-47.

[25] Rockafellar, R. T. and Royset, J. O. Superquantiles, and their applications to risk, random variables, and regression. Tutorials in operation research. Informs. (2013) 151-167.

[26] Rockafellar, R. T. and Uryasev, S. Optimization of conditional value-at-risk. Journal of Risk 2 (2000) 21-41.

[27] Trindade, A. A., Uryasev, S., Shapiro, A. and Zrazhevsky, G. Financial prediction with constrained tail risk. Journal of Banking and Finance 31 (2007) 3524-3528. 